\title{
Enabling smartphone-based HD video chats by cooperative transmissions in CRNs
}

\author{
Tao Jing ${ }^{1}$, Xuewei Cui ${ }^{1}$, Wei Cheng ${ }^{2}$, Shixiang Zhu ${ }^{1}$, Yan Huo ${ }^{1}$ and Xiuzhen Cheng ${ }^{3}$
}

\begin{abstract}
Smartphones have been equipped with the cameras that can shoot HD videos, and the video chat apps such as Skype are becoming popular. We can, therefore, intuitively predict the trend that users are expecting to enjoy HD video chats via utilizing their smartphones. Most of the current Internet services, however, cannot support the live HD video transmissions because of their low uplink rate. In order to overcome this limit, we propose to offload the uplink transmissions to cooperative users via cognitive radio networks. Specifically, we first divide the video stream into several substreams according to the H.264/SVC standard and the cooperative users' uplink rates. Then, the cooperative users are selected by employing our proposed optimal multiple stopping method. Finally, the substreams are assigned to the selected cooperative users by a 0-1 Knapsack-based allocation algorithm. The simulation results demonstrate that our proposed scheme can successfully support 720P HD video chats.
\end{abstract}

Keywords: Video chat; Cooperative relay selection; Optimal multiple stopping theory

\section{Introduction}

High-definition cameras are currently available on popular smartphones. These cameras have been physically ready to support $\mathrm{HD}$ video shootings (such as $720 \mathrm{P}$ and $1,080 \mathrm{P}$ ). According to [1], 720P and 1,080P videos require the transmission speeds of 6 and 12 Mbps, respectively. However, most of the popular Internet services are mainly optimized for downlink transmission. The uplink transmission rate supported by most popular Internet access networks is only $2 \mathrm{Mbps}$, and the typical upload speed on 3G/4G approximately ranges from 0.45 to $1.93 \mathrm{Mbps}$ [2]. As a result, a single user's Internet upload speed is generally not enough to support live HD video transmission. Therefore, an open problem is how could users enjoy the HD video chats without upgrading their Internet services, which may be too expensive and/or not necessary for other applications.

In order to enable the smartphone-based HD video chat, we propose to utilize cooperative users to help with the uplink transmission in cognitive radio networks (CRNs). The challenge of this task is how to efficiently select appropriate $\mathrm{CR}$ relays for cooperative uplink transmission

\footnotetext{
*Correspondence: wcheng3@vcu.edu

2 Department of Computer Science, Virginia Commonwealth University, 401 West Main Street, 23284 Richmond, VA, USA

Full list of author information is available at the end of the article
}

as the real-time requirement of video chat is very strong. In other works, the sender does not have enough time to look at all the candidate relays to select the best subset for the transmission. The sender has to do quick decisions on relay selection while guaranteeing the HD video transmission with a reasonable low cost. Therefore, our objective is to design an efficient relay selection method, which can support HD video chat with the lowest cost, and a load assignment method, which can optimally distribute the video data among the selected relays. Specifically, the major work and the contributions can be summarized as the following:

- Overall, we propose a scheme to support smartphone-based HD video chat without upgrading the user's internet service by utilizing the cooperative CR users' unlink resource. Simulations verify that our proposed scheme can successfully support 720P HD video chat.

- Intuitively, the selected relays can use more time to forward packets if the relay selection time can be reduced. According to the requirement of $\mathrm{HD}$ video transmission rate and the cooperative CR users' uplink rate, given a number of time slots, we analyze the relationship between the relay selection time and

\section{Springer}

(c) 2015 Jing et al: licensee Springer. This is an Open Access article distributed under the terms of the Creative Commons Attribution License (http://creativecommons.org/licenses/by/4.0), which permits unrestricted use, distribution, and reproduction in any medium, provided the original work is properly credited. 
the relay forwarding time so that the requirement can be satisfied.

- To the best of our knowledge, this is the first work to formulate an optimal multiple stopping model to solve the problem of relay selection for supporting $\mathrm{HD}$ video chat via cooperative transmission. We derive the multiple optimal stopping rules by jointly considering the instantaneous reward (of selected relays) and the expected sum reward (of the unobserved candidate relays). The proposed selection method can select the relays, whose instantaneous reward is at least the same as the expected sum reward.

- Extensive simulations have been conducted to investigate the impact of the parameters on the performance of the proposed scheme.

The rest of this paper is organized as follows. The most related work is summarized in Section 2. The overview of H.264/SVC, the network model, and the adopted relaying framework are illustrated in Section 3. Section 4 introduces our optimal stopping policy-based cooperative relay selection scheme and the packets assignment algorithm. The results of the performance evaluation are reported in Section 5. The paper is concluded in Section 6.

\section{Related work}

In recent years, researchers have shown a great interest in HD video technology. Jansen, 2011 [3] studies video-conferencing system for home. Lu, et al. 2010 [4] studies and compares mechanisms and performance of the existing video conference systems. Mirta, et al. 2010 [5] introduces a HD video broadcasting scheme by using scalable video coding so that the devices under various network environments can obtain the video with different resolutions. All of these work are studied under ideal network situations. However, for smartphone users, their uplink rate is generally not enough for HD transmission.

Several current video coding standards such as H.264/AVC, Dirac, AVS China, and VC-1 are introduced in [6]. Schwarz, et al. 2012 [7] provides the comparison of the coding efficiency for these video coding standards. Schwarz, et al. 2007 [8] gives a detailed overview of the scalable video coding extension of the H.264/AVC standard. In our work, we consider H.264/SVC as the coding standard utilized by HD video chat app.

An overview of existing cooperative relaying selection schemes is provided in [9]. They require channel-related information from all the candidate relay nodes, which is inefficient when the number of candidate relays is large or the time for relay selection is limited [10]. For example, channel state information and SNR are required by the relay selection approaches proposed in $[11,12]$, respectively. Moreover, [12] needs to compute the SNR thresholds for all candidate relays.

Jing, et al. 2013 [13] studies the relay selection problem in cognitive networks by applying optimal stopping theory. This approach does not look at the information from all the candidate relay nodes as it scans the candidate secondary user (SU) relays one by one and stops when a suitable relay is identified. As a result, this relay selection process is efficient for single relay selection. As a comparison, our work is the first one to apply optimal multiple stopping theory for multiple relay selection in cognitive radio networks, to our best knowledge. We design an optimal multiple stopping rule to find out the relays with good performance and within a short observation time.

\section{Preliminaries}

\subsection{Overview of H.264/SVC}

H.264/SVC standard, an extension of the H.264/AVC standard, is generally applied in HD video stream transmission. The speeds for 720P and 1,080P HD videos are 6 and $12 \mathrm{Mbps}$, respectively, according to [5]. An SVC video bit stream is essentially constructed with a base layer substream and more than one scalable enhancement layer sub-stream referring to different video layers. If part of the scalable stream is lost, the rest of the sub-stream forms a new valid bit stream with a certain bit-rate and reduced quality level which can be decoded by the target decoder. Hence, SVC can be applied in lossy transmission environments where graceful degradation of rate, format, and power adaptation exists. It is believed that H.264/SVC allows $20 \%$ packet loss in the network without affecting the quality [14].

Compared to the bit stream derived by dropping packets, a sub-stream can tolerate a lower temporal resolution (lower frame rate), lower spatial resolution (smaller screen), or lower quality video signal, which are named by these three modalities of scalability: temporal scalability, spatial scalability, and quality scalability.

\subsection{System model}

We consider a simple time-slotted cooperative transmission model depicted in Figure 1 which consists of a pair of primary users and a number of secondary users. To implement a HD video chat, a primary user (PU) transmitter, denoted by $P_{t}$, transmits its HD video stream to a primary user receiver, denoted by $P_{r}$, with the assistance of multiple cooperative users, who are the secondary users (SUs). The $n$ secondary users, represented by $S_{i}$, $i=1,2, \cdots, n$, have the ability to help transmit packets for the primary users, which are called candidate relays. When $P_{t}$ needs to transmit packets to $P_{r}, m$ free secondary users, which have favorable channel condition, can be selected as relay nodes by the PU transmitter. The $m$ 


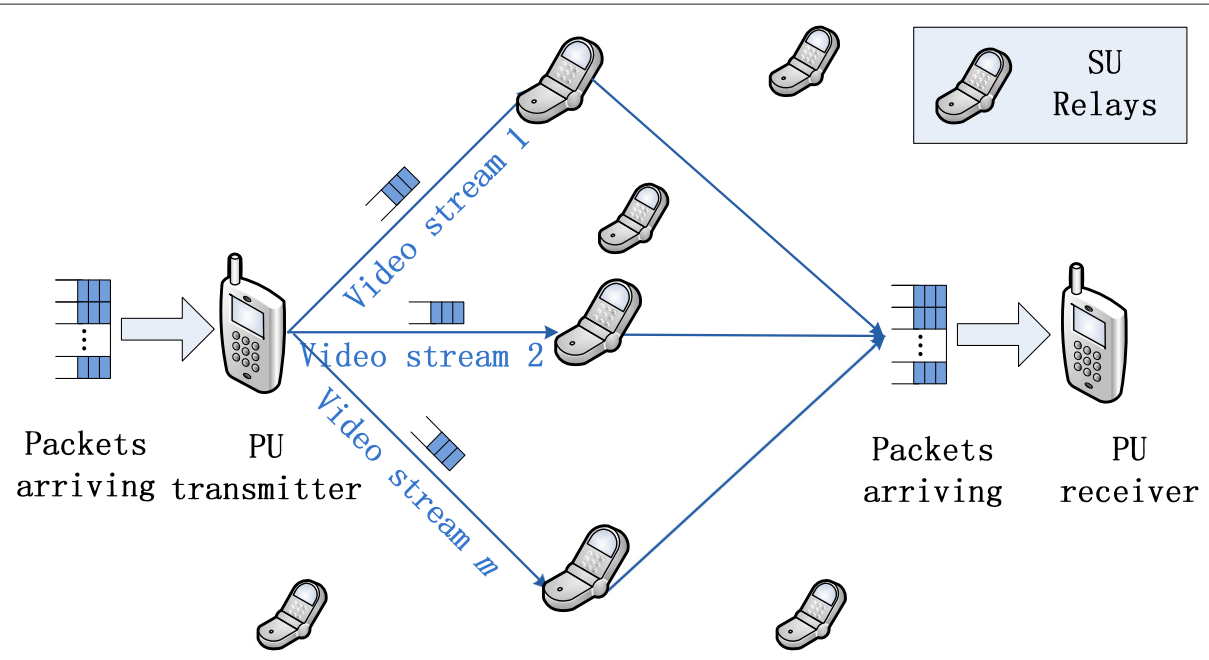

Figure 1 Cooperative transmission model.

secondary users finally selected by the PU transmitter are called cooperative relays.

It is assumed that the multiple cooperative relay selection is performed at each time slot. The duration of a time slot is $T=\frac{D_{\mathrm{hd}}}{R_{\mathrm{hd}}}$, where $D_{\mathrm{hd}}$ represents the amount of the packets to be transmitted for HD video and $R_{\text {hd }}$ represents the required transmission rate for $\mathrm{HD}$ video.

As illustrated in Figure 2, each time slot $T$ is partitioned into three components $T_{\mathrm{se}}, T_{\mathrm{sr}}$, and $T_{\text {rd }}$. Let $\tau$ be the time needed for observing a candidate relay. We assume that $\tau$ is identical for different SUs and for different time slots. Denoted by $S=\left\{s_{1}, s_{2}, \cdots, s_{n}\right\}$ an observation order/sequence, which is a permutation of the SU candidate relays index set $\{1,2, \cdots, n\}$. At the beginning of a time slot, $P_{t}$ starts to observe the $\mathrm{SU}$ candidate relay nodes sequentially according to the observation sequence. If the reward of the $k$ th observation satisfies a specific criterion, $P_{t}$ stops at the $k$ th SU candidate relay node and then continues to observe candidate relay nodes for the following stops. The whole observation ends after $P_{t}$ stops $m$ times.
$T_{\text {se }}$ represents the time of selecting relays in each time slot. After the cooperative relay selection process, $P_{t}$ transmits packets to relay node $i$ in $T_{i}$. The total time for transmission between PU transmitter and the relay nodes is denoted by $T_{\mathrm{sr}}$, which is the sum of $T_{1}, T_{2}, \cdots, T_{m}$. Then, the $m$ cooperative relays forward the video stream packets to $P_{r}$ simultaneously in $T_{\mathrm{rd}}$.

From Figure 2, we can see that:

$$
T=T_{\mathrm{se}}+T_{\mathrm{sr}}+T_{\mathrm{rd}}
$$

$T$ and $T_{\text {sr }}$ are given by:

$$
T=\frac{D_{\mathrm{hd}}}{R_{\mathrm{hd}}}, T_{\mathrm{sr}}=\frac{D_{\mathrm{hd}}}{R_{\mathrm{sr}}}
$$

where $R_{\mathrm{sr}}$ represents the transmittion rate between the PU transmitter and the cooperative relay node. Then we can get:

$$
T_{\mathrm{se}}+T_{\mathrm{rd}}=T-T_{\mathrm{sr}}=\frac{D_{\mathrm{hd}}}{R_{\mathrm{hd}}}-\frac{D_{\mathrm{hd}}}{R_{\mathrm{sr}}}
$$

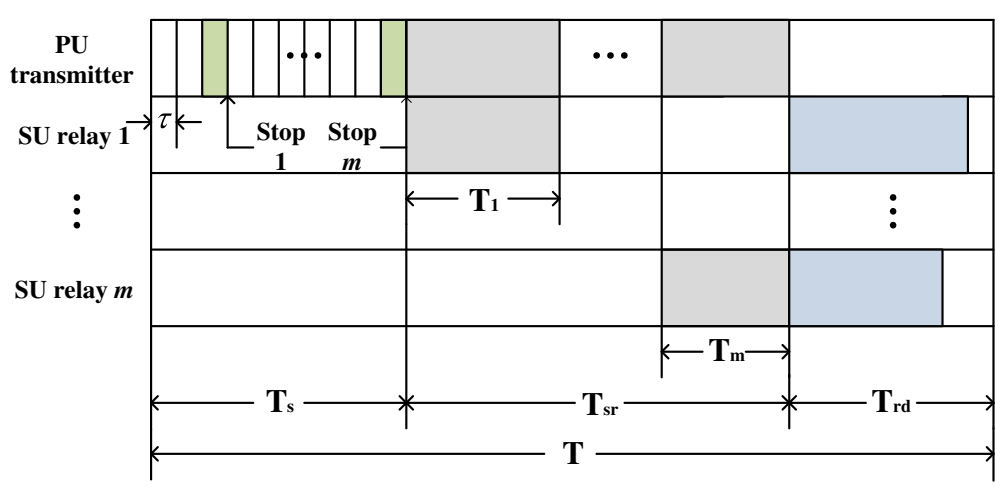

Figure 2 The time slot structure. 
During $T_{\mathrm{rd}}$, the cooperative relays forward the video packets to the PU receiver simultaneously. When there are $m$ relays forwarding packets, the estimated value of $T_{\mathrm{rd}}$ is given by:

$$
T_{\mathrm{rd}}^{*}=\frac{D_{\mathrm{hd}}}{m R_{\mathrm{rd}}^{*}}
$$

where $R_{\text {rd }}^{*}$ denotes the expected rate of a relay node in a flat Rayleigh fading channel. Then, we can get the estimated observation time and the estimated size of observation sequence $n$ below:

$$
\begin{aligned}
T_{\mathrm{se}}^{*} & =\frac{D_{\mathrm{hd}}}{R_{\mathrm{hd}}}-\frac{D_{\mathrm{hd}}}{R_{\mathrm{sr}}}-\frac{D_{\mathrm{hd}}}{m R_{\mathrm{rd}}^{*}} \\
n & =\frac{T_{\mathrm{se}}^{*}}{\tau}
\end{aligned}
$$

Obviously, the size of observation sequence should be larger than the number of cooperative relays. Since the condition that observation time $T_{\mathrm{se}}$ is larger than zero should be satisfied, we estimate the value of $m$ with a variable parameter $K$ as:

$$
m^{*}=\min \left\{m \mid n>m+K, T_{\text {se }}^{*}>0\right\}
$$

There are two types of relaying techniques: decode and forward (DF) and amplify and forward (AF) $[15,16]$. In $\mathrm{DF}$, a relay node first decodes the encoded data from the source node and then recodes before forwarding it to the destination node [17]. Different from the DF technique, a relay node in AF simply amplifies the signal of the received packets and then delivers them $[18,19]$. In this paper, we adopt AF to illustrate our designs.

This cooperative video stream transmission scheme can be divided into three steps: (i) the video source selects a group of cooperative users that have the independent Internet access and are willing to help; (ii) after encoding the video in H.264-SVC, the video source distributes the video frames to the cooperative users via CR channels; and (iii) the cooperative users forward the video frames to the destination via their own Internet access networks, then, the video destination can get the HD video after decoding it successfully. Undoubtedly, the whole process is transparent to the destination. The procedure of HD video transmission is depicted in Figure 3.

\section{Optimal multiple stopping policy}

\subsection{Problem formulation}

In this paper, we formulate the cooperative transmission problem as multiple stopping problem in CRNs. In order to select $m$ relays for cooperative transmissions, the PU transmitter observes multiple SU candidate relays one by one in an observation sequence. After contacting with a candidate relay, the PU decides whether to make a stop to select the current relay as a cooperative relay. The observation comes to the end if the PU finishes selecting $m$ relays. When PU makes a decision, it considers the reward in the sum case. In other words, we assume the PU has selected $l$ relays before observing $i$ th relay, if PU selects the current relay, we can get a sum reward denoted by $y_{i}+V_{i}^{m-l-1}$, where $y_{i}$ represents the instantaneous reward of the $i$ th relay and $V_{i}^{m-l-1}$ represents an expected reward of the $m-l-1$ relays to be selected from the remaining candidate relays; if PU does not select the current relay, we can get an expected sum reward of $m-l$ relays to be selected from the remaining candidate relays $V_{i}^{m-l}$. If the sum reward with selecting the current relay is larger than the one without selecting the current relay, the PU makes a stop and vice versa. Therefore, the multiple relay selection problem can be further formulated as a sequential decision problem which can be solved by the optimal multiple stopping theory.

We first introduce a definition of multiple stopping theory in the sum case. We, then, formulate our cooperative relay selection problem as an optimal multiple stopping problem in the sum case.

Definition 1. A multiple stopping rule problem in the sum case is defined by two parts:

- A sequence of random variables, $X_{1}, X_{2}, \cdots$, which have a known joint distribution

- A sequence of sum reward function $z_{0}^{l}=y_{0}+V_{0}^{m-l}$, $z_{1}^{l}\left(x_{1}\right)=y_{1}\left(x_{1}\right)+V_{1}^{m-l}, \cdots, z_{j}^{l}\left(x_{1}, x_{2}, \cdots, x_{j}\right)=$ $y_{j}\left(x_{1}, x_{2}, \cdots, x_{j}\right)+V_{j}^{m-l}$, where $y_{j}\left(x_{1}, x_{2}, \cdots, x_{j}\right)$ represents the instantaneous reward of $j$ th relay, $V_{j}^{m-l}$ represents the expected sum reward of $m-l$ relays to be selected in the remaining candidate relays.

The objective is to find out the variables in the sequence such that the sum reward function is maximized.

In order to further investigate the channel quality in our cooperative relay selection problem, we assume that the underlying channel is a flat Rayleigh fading channel [20], in which the instantaneous signal-to-interferenceplus-noise ratio (SNR) is received by the destination with

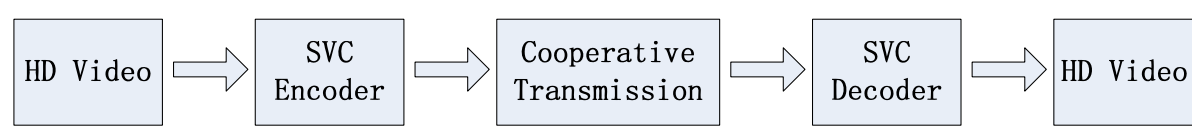

Figure 3 The procedure of HD video transmission. 
an exponential distribution having a probability density function (PDF) $f(\gamma)=\frac{1}{\bar{\gamma}} e^{-\frac{\gamma}{\bar{\gamma}}}$, where $\bar{\gamma}$ denotes the average SNR in the channel model. Then, we can model the Rayleigh fading channel as a finite state Markov chain (FSMC) as proposed in [6]. In the FSMC, we partition the SNR into U intervals and then divide SNR into a finitestate space. Thus, the SNR thresholds are denoted by $\Upsilon=$ $\left\{\gamma_{1}=0, \gamma_{2}, \cdots, \gamma_{U}, \gamma_{U+1}=\infty\right\}$. If an instantaneous SNR $\Gamma$ is in $\left[\gamma_{u}, \gamma_{u+1}\right)$, the channel of the SU candidate relay is said to be in state $s_{u}$. When the PU pair observes the channel of the candidate relay, the probability of the SU being in state $s_{u}$ for the channel can be given by:

$$
q_{u}=\int_{\gamma_{u}}^{\gamma_{u+1}} f(\gamma) d \gamma=e^{-\frac{\gamma_{u}}{\bar{\gamma}}}-e^{-\frac{\gamma_{u+1}}{\bar{\gamma}}}, u=1, \cdots, U
$$

In our cooperative transmission problem, the achievable transmission rate is viewed as a metric for the channel quality in wireless communications. Let $r_{k}$ denote the achievable transmission rate between the PU pair and the SU candidate relay node $k$. According to the Shannon's theorem [21], $r_{k}$ is calculated as follows:

$$
r_{k}=W \log \left(1+\gamma_{k}\right)
$$

where $W$ denotes the bandwidth of the spectrum. Thus, the corresponding date rate can be denoted as $R=$ $\left\{r_{1}, r_{2}, \cdots, r_{U}\right\}$. We can also model the transmission rate as a discrete random variable, which has a same distribution as the channel state:

$$
\operatorname{Pr}\left\{R=r_{u}\right\}=q_{u}, u=1,2, \cdots, U
$$

The PU pair acquires the achievable transmission rate of the channel between itself and the SU candidate relay by executing the observation in relay selection. The process of observation is similar to the request-to-send (RTS)/clear-to-send (CTS) access mechanism designed for the 802.11 technique [22,23]. At each observation step, the PU transmitter sends a RTS frame to the candidate relay. Upon receiving of a RTS frame, the candidate relay returns a CTS frame, which includes the information for calculating the achievable rate. We define that $X_{k}=R_{k}$ is the valid transmission rate of the $k$ th observation step. Then, the distribution of $X_{k}$ can be calculated as follows:

$$
\begin{aligned}
& \quad p_{u}=\operatorname{Pr}\left\{X_{k}=x_{u}=r_{u}\right\}=q_{u} \\
& \text { for } 1 \leq u \leq U, 1 \leq k \leq n
\end{aligned}
$$

Then, we derive the instantaneous reward function denoted by $Y_{k}$ based on the valid transmission rate and two scaling factors. We denote $e(k, l)$ as the scaling function if the PU pair stops at the $k$ th observed candidate relay node with $l$ selected relays:

$$
e(k, l)=1-\frac{k(m-l)}{M}
$$

where $m$ also means the stopping times for the whole observation and $l$ also means the times we have stopped. $M$ is a variable parameter. From (12), we can see that $e(k, l)$ is an increasing function of $k$ and a decreasing function of $l$. In other words, a larger number of SU candidate relay nodes, which the PU transmitter has observed, means the lower efficiency of the cooperative relay selection process. More relays, which have been selected by the PU transmitter, means the less number of stops remains. As a result, the efficiency of the cooperative relay selection process becomes higher. Accordingly, the reward after the $k$ th observation attempt with $l$ stops is given by:

$$
Y_{k}=e_{k} X_{k}
$$

After deriving the reward function, we summarize the optimal multiple stopping problem in cooperative relay selection as follows: the PU pair receives the reward $Y_{k}$ after the $k$ th observations, then the PU transmitter makes a decision on whether to make a stop at the current candidate relay. The PU transmitter finally stops the observation till $m$ cooperative relays are all selected. Note that no recall is allowed since the channel quality is changing rapidly in cognitive radio networks due to the complicated conditions such as the mobility of the users.

\subsection{Optimal multiple stopping rule}

In this subsection, we solve the multiple stopping problems in the sum case by deriving the optimality equations. We also prove that the proposed rule is better than the random stopping rule, in which the PU stops $m$ times randomly.

We denote $F_{i}$ as the probability distribution function of the instantaneous reward $Y_{i}$ after the $i$ th observation. For the finite payoff reward sequence $Y_{1}, \cdots, Y_{n}$ that are independent and identically distributed as depicted in Section 4.1, the optimality equations can be stated as follows for the $m$-stopping problems. In case $m=1$, a well-known recursive solution of stopping problem is defined by:

$$
\begin{aligned}
V_{n} & :=-\infty \\
V_{i} & :=E\left[Y_{i+1} \vee V_{i+1} \mid F_{i}\right]
\end{aligned}
$$

where $V_{i}^{m}$ represents the expected sum reward of continue observing for $m$ stopping times after $i$ th observation and $\vee$ denotes the maximum. We need a version of this classical result for stopping times larger than a given stopping time $C$.

Proposition 1. Recursive solution of one-stopping problems.

(a) For any time point $0 \leq k \leq n-1$, the stopping time

$$
T(k):=\min \left\{k<i \leq n: Y_{i}>V_{i}\right\}
$$


is optimal in the sense that for any stopping time we have

$$
E\left[Y_{T(k)} \mid F_{k}\right]=V_{k} \geq E\left[Y_{T} \mid F_{k}\right]
$$

(b) For any stopping time $C$, the stopping time

$$
T(C):=\min \left\{C<i \leq n: Y_{i}>V_{i}\right\}
$$

is optimal in the sense that for any stopping time $T$ with $C<T$ conditionally on $\{C<n\}$ and $C=T$ conditionally on $C=n$, we have

$$
E\left[Y_{T(C)} \mid F_{C}\right]=V_{C} \geq E\left[Y_{T} \mid F_{C}\right]
$$

For $m$-stopping problems, the following variant of Proposition 1 will also be needed. Let $C$ be a stopping time, let $B$ be measurable with the same probability distribution with $Y_{i}$ and $h$ be measurable with $\operatorname{Eh}\left(Y_{i}, B\right)^{+}<\infty$. We can also define recursively for $b$ :

$$
\begin{aligned}
V_{n}(b) & :=h\left(Y_{n}, b\right) \\
V_{i}(b) & :=E\left[h\left(Y_{i+1}, b\right) \vee V_{i+1}(b) \mid F_{i}\right]
\end{aligned}
$$

Then the stopping time:

$$
T(C, B):=\min \left\{C<i \leq n: h\left(Y_{i}, B\right)>V_{i}\left(B_{i}\right)\right\}
$$

is optimal in the sense that for any further stopping time $T$ with $C<T$ conditionally on $C<n$ and $C=T$ conditionally on $C=n$, we have:

$$
E\left[h\left(Y_{T(C, B), B)} \mid F_{C}\right]=V_{C}\left(B_{C}\right) \geq E\left[h\left(Y_{T}, B\right) \mid F_{C}\right]\right.
$$

The idea of solving $m$-stopping problem is based on the one for solving the one-stopping problem. The $l$ th stopping time $T_{l}$ should be $i$, if the total expected reward by taking $i$ as a stop is larger than the one, where $i$ will not be a stop. We define the thresholds $V_{n-m+1}^{m}, V_{i}^{0}$ and $V_{i}^{m}$ by:

$$
\begin{aligned}
V_{n-m+1}^{m} & :=-\infty \\
V_{i}^{0} & :=0 \\
V_{i}^{m} & :=E\left[\left(Y_{i+1}+V_{i+1}^{m-l}\right) \vee V_{i+1}^{m} \mid F_{i}\right]
\end{aligned}
$$

From (26), we know that $V_{i+1}^{m-1}$ and $V_{i+1}^{m}$ can be calculated by backwards induction. We compute $V_{i}^{m}$ as follows:

$$
\begin{aligned}
V_{i}^{m} & =E\left[\left(Y_{i+1}+V_{i+1}^{m-1}\right) \vee V_{i+1}^{m} \mid F_{i}\right] \\
& =E\left\{\left[e(k, l) X_{i+1}+V_{i+1}^{m-1} \vee V_{i+1}^{m}\right] \mid F_{i}\right\} \\
& =\sum_{\alpha}\left[e(k, l) x_{\alpha}+V_{i+1}^{m-1}\right] p_{\alpha}+\sum_{\beta} V_{i+1}^{m} p_{\beta}
\end{aligned}
$$

where $\alpha \in\left\{k \mid e(k, l) x_{k}+V_{i+1}^{m-1}>V_{i+1}^{m}, k=0,1,2, \cdots, U\right\}$, $\beta \in\left\{k \mid e(k, l) x_{k}+V_{i+1}^{m-1}<V_{i+1}^{m}, k=0,1,2, \cdots, U\right\}$, subject to:

$$
\left\{\begin{array}{l}
0 \leq \alpha \leq U \\
0 \leq \beta \leq U \\
\alpha+\beta=U
\end{array}\right.
$$

Then, we can get the sum reward function:

$$
z_{i}^{l}\left(x_{1}, x_{2}, \cdots, x_{i}\right)=y_{i}\left(x_{1}, x_{2}, \cdots, x_{i}\right)+V_{i}^{m-l}
$$

where $y_{i}\left(x_{1}, x_{2}, \cdots, x_{i}\right)$ denotes the instantaneous reward after the $i^{t h}$ observation. It is optimal to stop if $z_{i}^{l}\left(x_{1}, x_{2}, \cdots, x_{i}\right)$ is larger than $V_{i}^{m-l+1}$.

The related threshold stopping times are defined recursively for $1 \leq m \leq n, 0 \leq k \leq n-m$ by:

$T_{1}^{m}(k):=\min \left\{k<i \leq n-m+1 \mid z_{i}^{1}\left(x_{1}, x_{2}, \cdots, x_{i}\right)>V_{i}^{m}\right\}$

$$
\begin{aligned}
T_{l}^{m}(k) & :=\min \left\{T_{l-1}^{m}(k)<i \leq n-m+l \mid z_{i}^{l}\left(x_{1}, x_{2}, \cdots, x_{i}\right)\right. \\
& \left.>V_{i}^{m-l+1}\right\}, 2 \leq m \leq n
\end{aligned}
$$

As in the one-stopping problem, it holds that $\left\{T_{i}^{m}(k)\right\}$ are optimal stopping times.

Proposition 2. For multiple stopping problem in the sum case, $\left\{T_{i}^{m}(k)\right\}$ are optimal m-stopping times in the sense that for all stopping times $k<T_{1}<\cdots<T_{m} \leq n$ it holds that:

$$
E\left[\sum_{l=1}^{m} Y_{T_{l}^{m}(k)} \mid F\right] \geq E\left[\sum_{l=1}^{m} Y_{T_{l}} \mid F\right]
$$

Proof 1. This proof is by induction in m. As depicted above, $\left\{Y_{i}\right\}$ are independent and identically distributed. In the case $m=1$, we can get:

$$
\begin{aligned}
E\left[Y_{T_{1}^{1}(k)} \mid F\right] & =V_{k}^{1} \\
& =E\left[\left(Y_{k+1}+V_{k+1}^{0}\right) \vee V_{k}^{1} \mid F\right] \\
& =E\left\{Y_{k+1} \vee \cdots E\left[Y_{n-1} \vee E\left(Y_{n}\right)\right] \mid F\right\} \\
& \geq(n-k) \cdot \frac{1}{n-k} E\left[Y_{i} \mid F\right]=E\left[Y_{T_{1}} \mid F\right]
\end{aligned}
$$

For the induction step $m \rightarrow m+1$, by induction hypothesis we obtain:

$$
\begin{aligned}
& E\left[Y_{T_{1}}+\cdots+Y_{T_{m+1}} \mid F\right] \\
& \quad=E\left[Y_{T_{1}}\left|F[+E]\left[Y_{T_{2}}+\cdots+Y_{T_{m+1}} \mid Y_{T_{1}}\right]\right| F\right] \\
& \quad \leq E\left[Y_{T_{1}^{m+1}(k)} \mid F\right]+E\left[Y_{T_{1}^{m}\left(T_{1}\right)}+\cdots+Y_{T_{m}^{m}\left(T_{1}\right)} \mid F\right] \\
& \quad=E\left[\sum_{l=1}^{m+1} Y_{T_{l}^{m+1}(k)} \mid F\right]
\end{aligned}
$$

This is maximized by choosing $T_{1}$ to be the optimal one-stopping time of the process $\left(Y_{i}+V_{i}^{m}\right)$. This 
optimal stopping time is given from the case $m=1$ by $T_{1}=T_{1}^{m+1}$. In consequence, the optimal stopping times for $1 \leq l \leq m$ are obtained by $T_{l}^{m}\left(T_{1}^{m+1}\right)=$ $T_{l+1}^{m+1}$.

Therefore, we propose the optimal multiple stopping rule in Algorithm 1. The PU observes an SU candidate relay node according to the observation sequence $S$ and obtains an instantaneous reward $y_{i}$ after the $i$ th observation (line 4). Then, the PU compares the sum reward $z_{i}^{l}$ with the expected sum reward $V_{i}^{m-l+1}$. If $z_{i}^{l} \leq V_{i}^{m-l+1}$, the PU continues to observe the next SU candidate relay. Otherwise, the PU pair makes a stop at the $i$ th relay. When $m$ cooperative relays are all selected, PU finishes the whole observation. Note that if the PU observes the $(n-m+l)$ th candidate relay with having selected $l$ cooperative relays, it has to select the rest of SU nodes as the cooperative relays for supporting the transmissions regardless of the value of the sum reward.

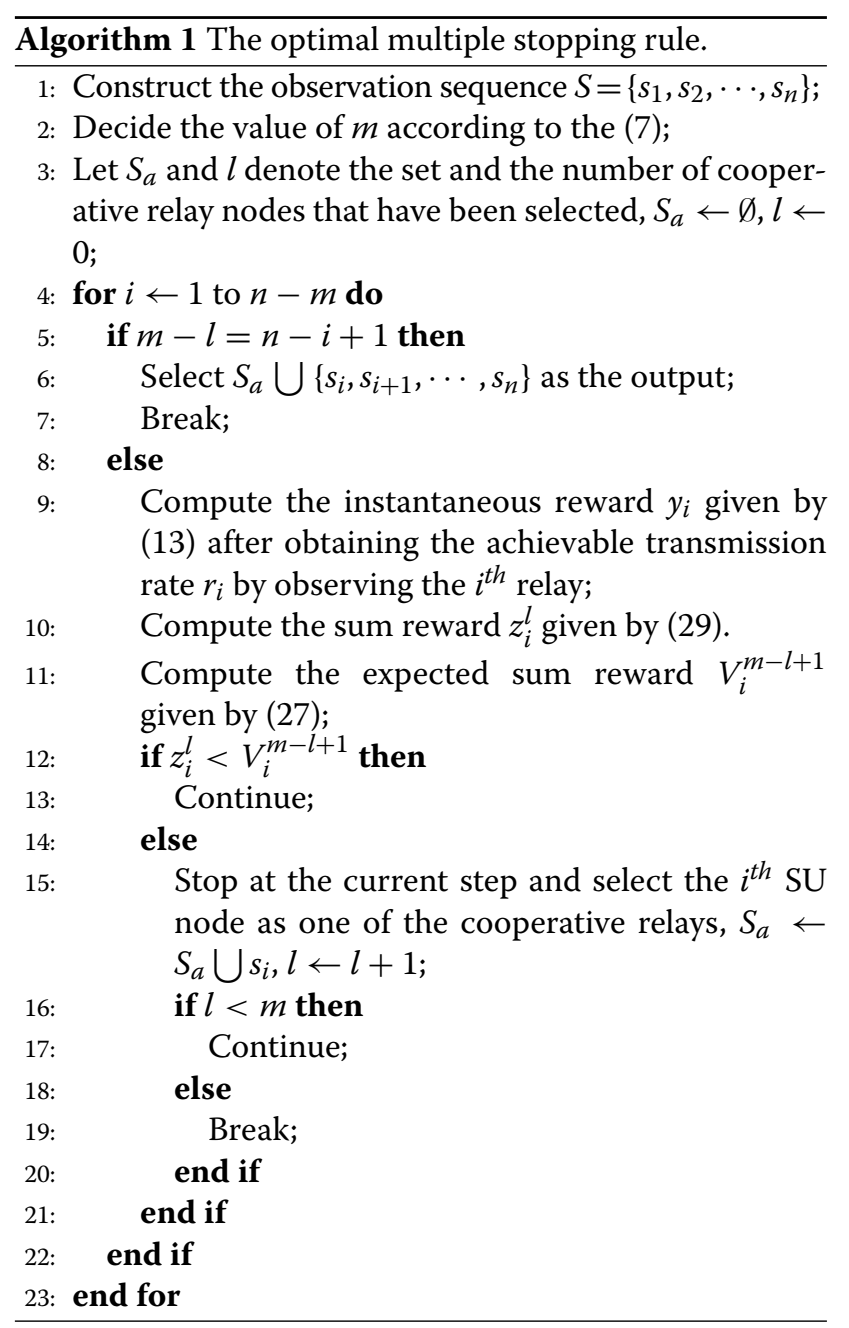

\subsection{Video frames allocation algorithm}

Since different cooperative relay nodes may have varied transmission rates, we target on maximizing the amount of transmission on each relay under certain rate limits. In this subsection, we intend to find an optimal video frames allocation algorithm by formulating it as 0-1 Knapsack problem.

The 0-1 Knapsack problem [24] is a subproblem of Knapsack problem, which is a problem in combinatorial optimization. We first give an brief definition of $0-1$ Knapsack problem, which is employed in our allocation model.

Definition 2. The 0-1 Knapsack problem is defined as follows: given a set of items, each with a weigh and a value, determine the number ( 0 or 1) for each item to include in a collection, so that the total weight is less than or equal to a given limit, and the total value is maximized.

The PU video source encoder divides the video stream into a base sub-stream and $M$ enhancement streams. Let $D=\left\{D_{1}, D_{2}, \cdots, D_{M}\right\}$ denote the set of divided packets, where $D_{k}(k=1,2, \cdots, M)$ represents the amount of a specific video packet. We allocate the packets to the selected cooperative relays one by one according to the descending order of their transmission rates. For the $i$ th cooperative relay $s_{i}$, we denote set $D^{i}=\left\{D_{1}^{i}, D_{2}^{i}, \cdots, D_{M_{i}}^{i}\right\}$ as the set of the amount of packets that have not been allocated.

We formulate the video frames allocated problem as follows. The PU selects $m$ cooperative relays with the rate $\left\{r_{1}, r_{2}, \cdots, r_{m}\right\}$ for forwarding packets to the PU receiver. Let $A_{i}, i=1,2, \cdots, m$, denote relay $s_{i}$ 'a available amount of transmissions, which is defined as the amount of packets that can be forwarded by $s_{i}$. In $T_{\text {rd }}$, the amount of packets, which relay $s_{i}$ can forward, is $r_{i} \cdot T_{\text {rd }}$. Therefore, we obtain $A_{i}=r_{i} \cdot T_{\text {rd }}$. Then, we define an available transmission amount of vector $A$ as $A=\left\{A_{1}, A_{2}, \cdots, A_{m}\right\}$.

In our allocation model, we take $D^{i}=\left\{D_{1}^{i}, D_{2}^{i}, \cdots, D_{M_{i}}^{i}\right\}$ as the weigh and the value for items of base layer and enhancement layers. We regard set $A=\left\{A_{1}, A_{2}, \cdots, A_{m}\right\}$ as the given limits depicted in Definition 2.

In 0-1 Knapsack problem, the weigh and the limit should be integers, we, therefore, construct a corresponding integer sets $D^{i *}=\left\{D_{1}^{i *}, D_{2}^{i *} \cdots, D_{M_{i}}^{i *}\right\}$ and $A^{*}=$ $\left\{A_{1}^{*}, A_{2}^{*}, \cdots, A_{m}^{*}\right\}$. Where $D_{k}^{i *}=\left\{D_{k}^{i}\right\}+1$ and $A_{i}^{*}=\left[A_{i}\right]$. Here, [.] represents the integral function. Note that after the allocation for a relay, the set $D^{i *}$ changes regularly.

The objective of our video frames allocation is to determine the subset $Q_{i} \subseteq\left\{1,2, \cdots, M_{i}\right\}$ such that:

$$
\max \sum_{t \in Q_{i}} D_{t}^{i *},
$$




$$
\text { subject to } \sum_{t \in Q_{i}} D_{t}^{i *} \leq A_{i}^{*} .
$$

For $1 \leq k \leq M_{i}, 0 \leq r \leq A_{i}^{*}$, we set:

$$
\begin{aligned}
& G(0, r)=0, G(k, 0)=0 \\
& \text { if } D_{k}^{i *}>r, G(k, r)=G(k-1, r) \\
& \text { if } D_{k}^{i *} \leq r, \\
& G(k, r)=\max \left\{D_{k}^{i *}+G\left(k-1, r-D_{k}^{i *}\right), G(k-1, r)\right\}
\end{aligned}
$$

From (38) and (39), we can see that there are only two choices for $\left\{D_{1}^{i *}, D_{2}^{i *} \cdots, D_{M_{i}}^{i *}\right\}$ to compute $G(k, r)$.

1. Leave $D_{k}^{i *}$ : the maximal amount that relay $i$ can forward from $\left\{D_{1}^{i *}, D_{2}^{i *} \cdots, D_{M_{i}}^{i *}\right\}$ with limit $r$ is $G(k-1, r)$.

2. Take $D_{k}^{i *}$ (only possible if $D_{k}^{i *} \leq r$ ): we gain $D_{k}^{i *}$ of transmission amount and the maximal amount that the relay can transmit from $\left\{D_{1}^{i *}, D_{2}^{i *} \ldots, D_{M_{i}}^{i *}\right\}$ with rate limit $r-D_{k}^{i *}$ is $G\left(k-1, r-D_{k}^{i *}\right)$. Totally, we get $D_{k}^{i *}+G(k-1)$.

To allocate packets for relay $i$, we first do the bottom-up computation using iteration by computing the table using (38) and (39) row by row as shown in Figure 4 .

The set $Q_{i}$ is derived by the process depicted in

\begin{tabular}{|c|c|c|c|c|c|c|}
\hline$G(k, r)$ & $r=0$ & 1 & 2 & 3 & $\ldots$ & $\mathbf{A}_{\mathbf{i}}^{*}$ \\
\hline$k=0$ & 0 & 0 & 0 & 0 & $\ldots$ & 0 \\
\hline 1 & - & & & & & $\longrightarrow$ \\
\hline 2 & - & & & & & $\longrightarrow$ \\
\hline$\vdots$ & - & & & & & $\longrightarrow$ \\
\hline$\overline{M_{i}}$ & - & & & & & $\longrightarrow$ \\
\hline
\end{tabular}
Algorithm 2, where $T_{x, y}$ represents the element in row $x$ column $y$ of the table. The video frames allocation algorithm which is based on the Knapsack problem is depicted in Algorithm 3.

Figure 4 Bottom-up computation table. Figure 4 has been moved to this new location, and the citation for Figure 4 has been added in the above paragraph.

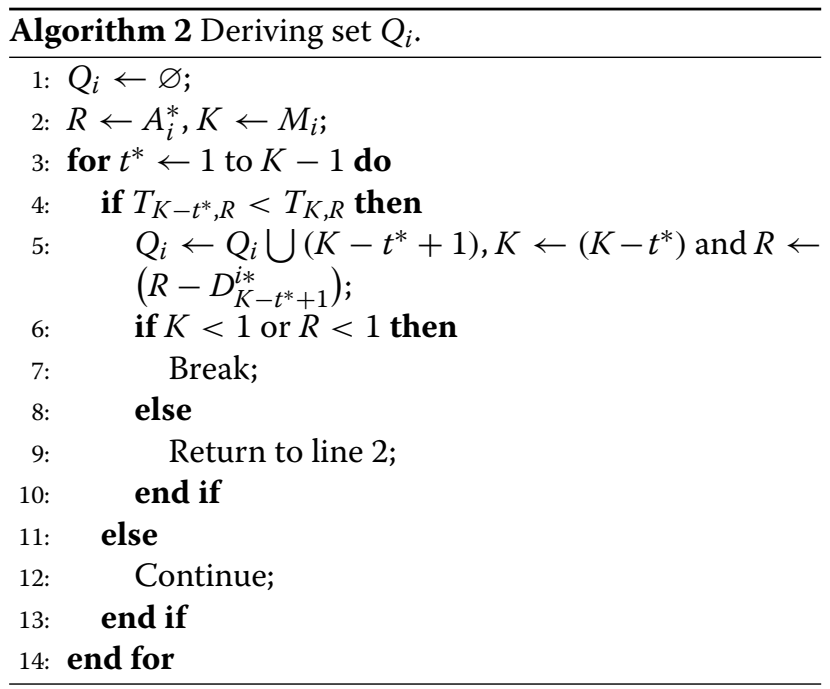

\section{Performance evaluation}

In this section, we evaluate the performance of our cooperative HD video transmission scheme by simulations. The video stream is divided into five parts: one base layer substream and four pieces of enhancement layer substream. It is assumed that the transmission rate $r_{k}$ of the $k$ th SU candidate relay node does not change within one slot. We divide the finite-state space of SNR received by the receiver into $U=30$ intervals. The bandwidth $W$ is set to be $1 \mathrm{MHz}$. The rate of PU source is set to be the average rate of channel. The controlling factor $M$ is set to 
be $10^{6}$. We set the transmission rate of $720 \mathrm{P}$ HD video as $6 \mathrm{Mbps}$. Each result reported in this section is the average of 100 instances.

To provide a deep insight into the feasibility of our cooperative HD video transmission scheme, we first make a comparison between our cooperative relay selection scheme and the random cooperative relay selection scheme. Then, we investigate the performance of our video frames allocation algorithm by comparing it with the random video frames allocation scheme. Moreover, we study the impact of the average SNR $\bar{\gamma}$ and the observation duration $\tau$ on the transmission performance.

\subsection{The comparison with random schemes}

In this subsection, we compare our cooperative relay selection scheme and video frames allocation algorithm with the corresponding random methods, respectively. We set the average SNR $\bar{\gamma}$ to be $30 \mathrm{~dB}$, the time duration $\tau$ to be $3 \mu$ s.

We change the transmission time $T$ from 0.4 to 0.85 $\mathrm{ms}$ and investigate the available cooperative transmission rate and the realistic cooperative transmission rate of schemes. Figure 5 reports the performance comparison of our cooperative relay selection scheme and the random relay selection scheme. We can see that selecting cooperative relays via optimal multiple stopping rule can achieve higher available cooperative transmission rate than selecting relays randomly. With the increasing of HD video transmission time, the available cooperative transmission rate of our scheme increases linearly, while the available cooperative transmission rate of random cooperative relay selection is distributed irregularly. From the equations in Section 4.1, we can see that with a specific SNR $\bar{\gamma}$, the number of candidate relays increases with $T$, which is explained in Section 5.2 in detail. So we have greater chance to find cooperative relays with good performance.

Then, we compare the proposed video frames assignment algorithm with the random allocation scheme. In this part, all the relay nodes are selected by our cooperative relay selection scheme. As Figure 6 shows, the realistic cooperative rate in the situation where applying the proposed video frames allocation is almost as same as the transmission rate for 720P HD video, which implies that our scheme can support HD video transmissions. We can also see that the realistic rate in the situation, where the packets are assigned randomly, is always lower than the transmission rate required by 720P HD video.

\subsection{The impact of the parameter $\bar{\gamma}$}

In this subsection, we investigate the impact of the parameter $\bar{\gamma}$ on the number of nodes, available cooperative transmission rate, and realistic cooperative transmission rate under the situation, where the average SNR $\bar{\gamma}$ changes regularly. We set the observation duration $\tau$ to be $3 \mu \mathrm{s}$. The transmission time $T$ is set to be $0.5,0.6$, and $0.7 \mathrm{~ms}$. We set $K=10$ in (7) when $\bar{\gamma} \geq 22 \mathrm{~dB}$ and $K=0$ when $\bar{\gamma}<22 \mathrm{~dB}$, respectively.

The relationship between $\bar{\gamma}$ with the the number of candidate relays and cooperative relays can be seen from Figure 7. We can see that the lager the average SNR $\bar{\gamma}$ is, the less cooperative relays we need. According to the

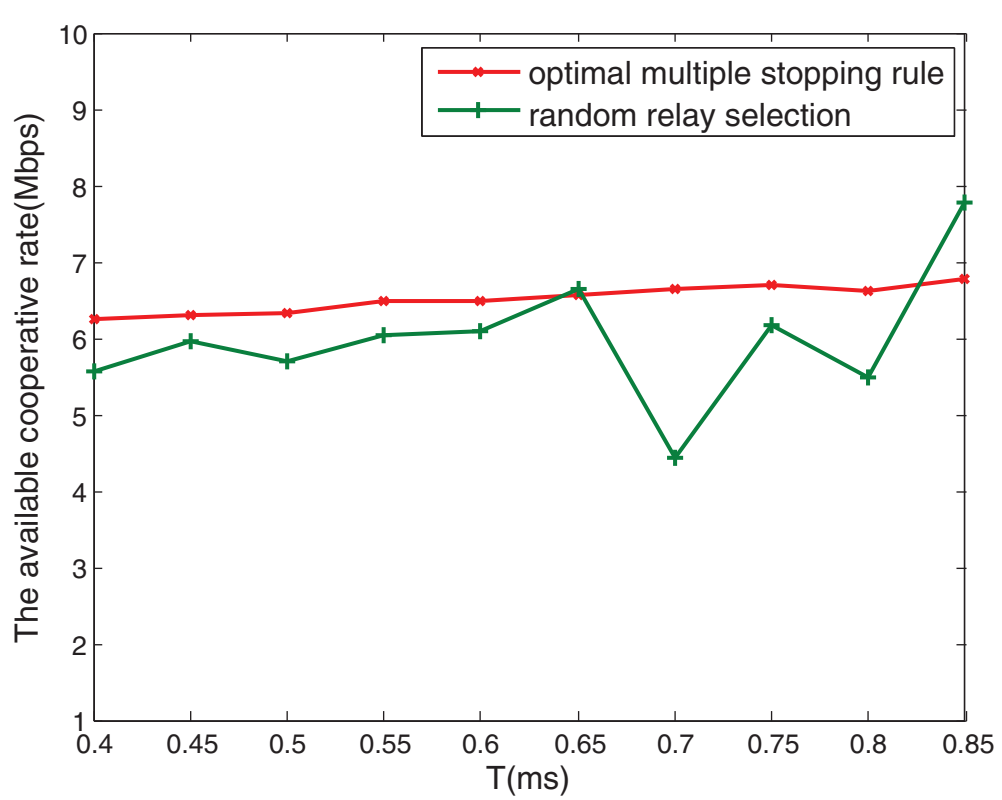

Figure 5 Our cooperative relay selection scheme vs. the random cooperative relay selection scheme. 


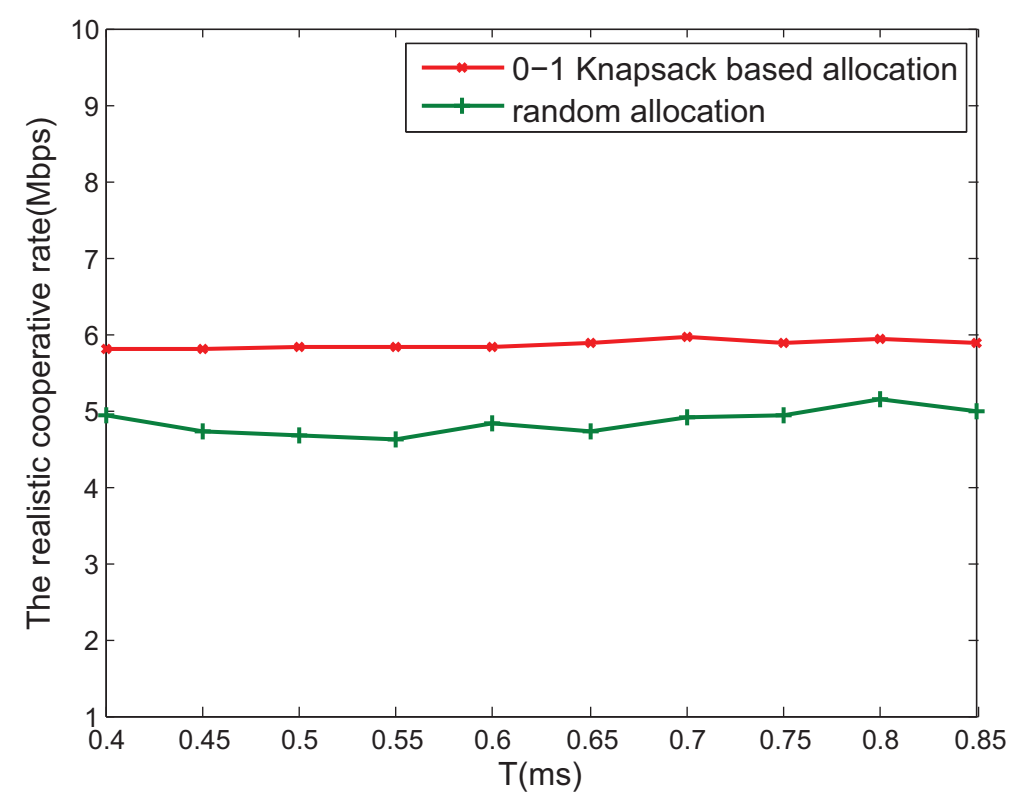

Figure 6 Assignment based on 0-1 Knapsack problem vs. random assignment.

analysis in Section 4.1, we can know that the observation time is decided by the number of cooperative relays and average SNR $\bar{\gamma}$, but the number of cooperative relays has a larger impact on the observation time for all candidate relays. Therefore, when the number of cooperative relays decreases one, the number of candidate relays that is in proportion to observation time reduces greatly; when the number of cooperative relays remains unchanged, the number of candidate relays impacted by $\bar{\gamma}$ increases linearity.

Note that when $\bar{\gamma}$ is below $22 \mathrm{~dB}$, the available transmission rate of candidate relays is low. As a result, we need to select more cooperative relays for cooperative transmissions. However, the number of candidate relays is less than the number of cooperative relays with the parameter $K$ in (7) is set to be 0 . Therefore, our

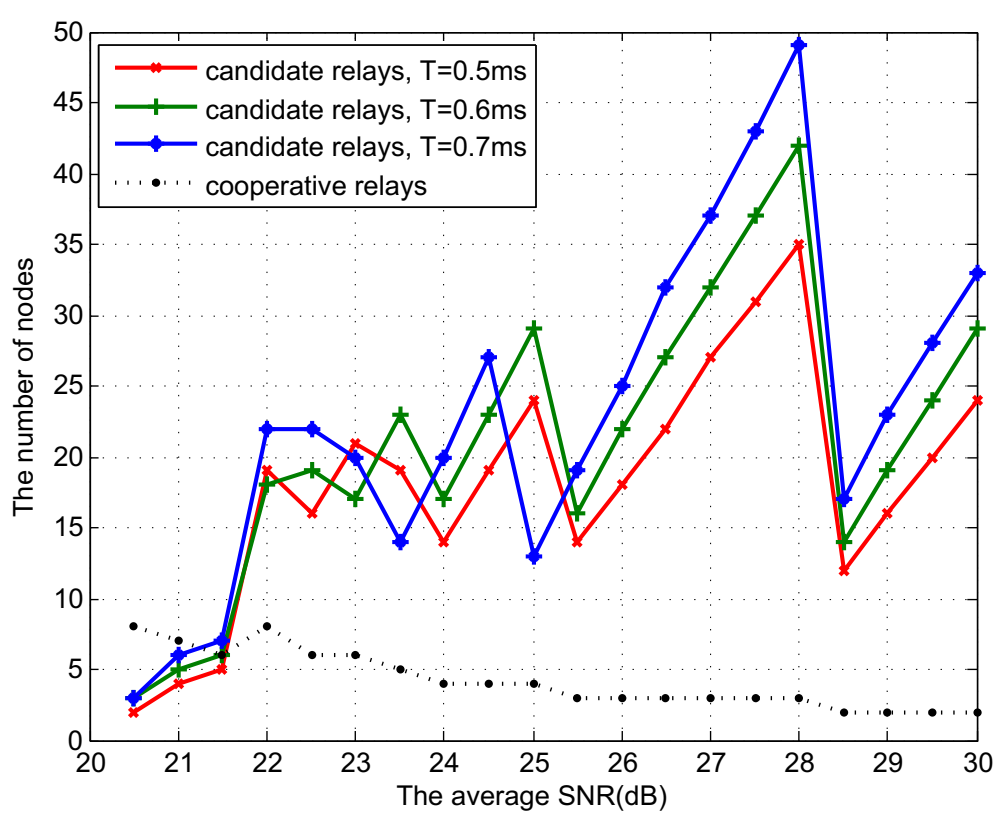

Figure 7 The number of relays vs. the parameter $\bar{\gamma}$. 


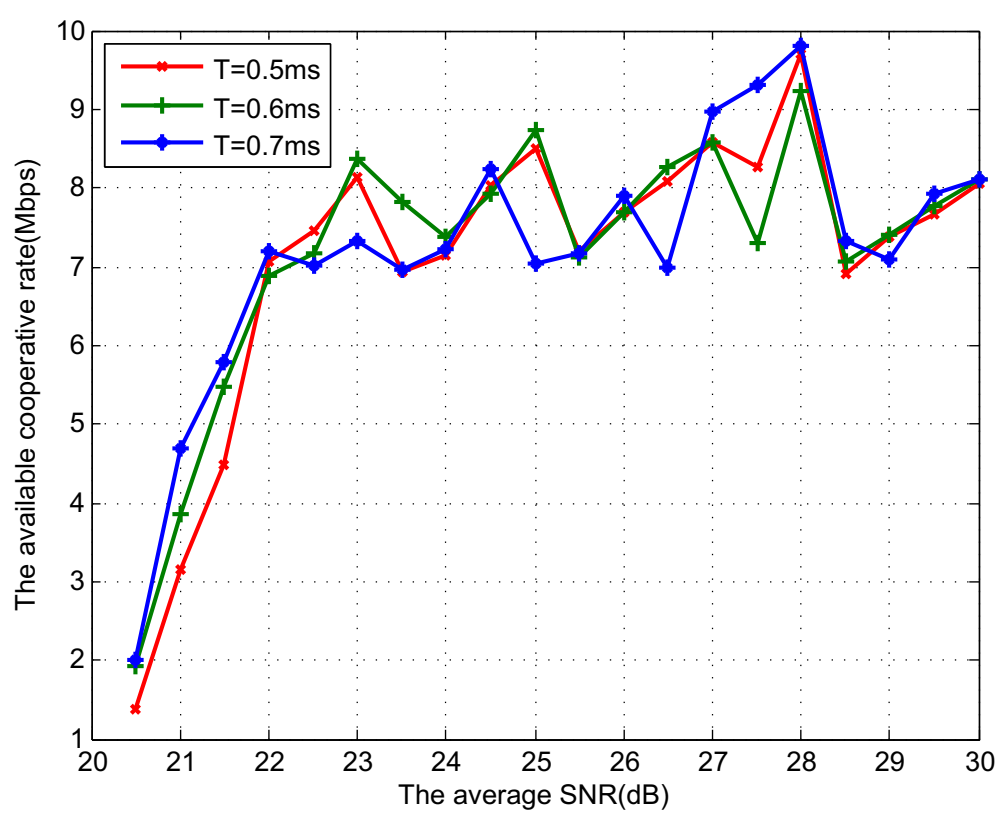

Figure 8 The available transmission amount vs. the parameter $\bar{\gamma}$.

proposed scheme is infeasible when the average SNR $\bar{\gamma}$ is low.

Form Figure 8, we can see that the available cooperative transmission rate increases with rising average SNR. We know from Figure 7 that the higher average SNR results in the larger number of candidate relays when the number of cooperative relays remains unchanged. With the increasing number of candidate relays, the PU transmitter has higher probability to choice relays with better performance. Therefore, the available cooperative transmission rate increases. Similar results can be obtained in Figure 9. With the increasing of $\bar{\gamma}$, the realistic cooperative transmission rate increases at the beginning, then stays in a stable state that can satisfy the transmission rate for supporting 720P HD video.

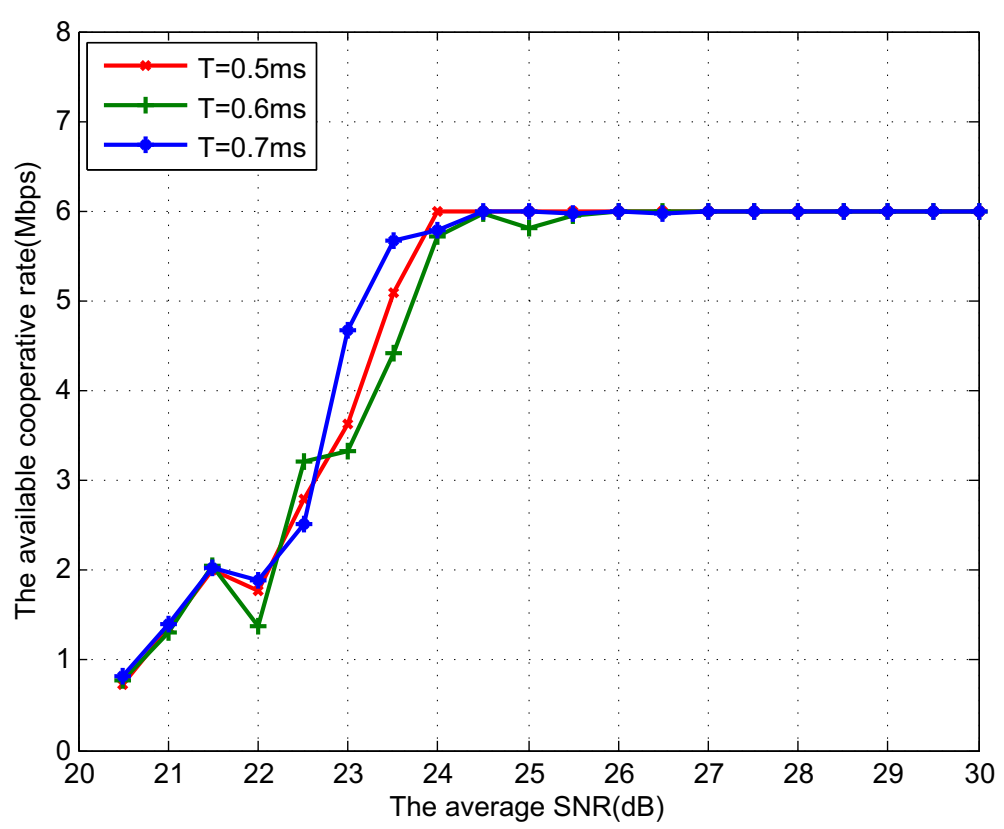

Figure 9 The realistic transmission amount vs. the parameter $\bar{\gamma}$. 


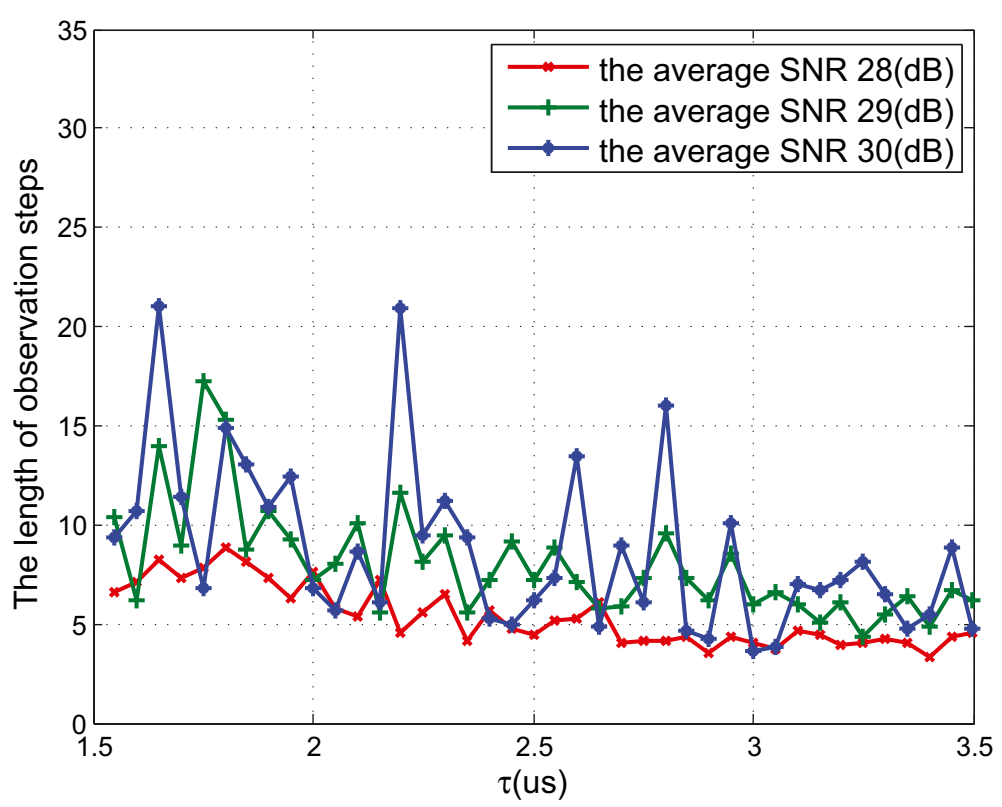

Figure 10 The length of observation steps vs. time for one observation.

\subsection{The impact of observation duration $\tau$}

In this subsection, we investigate the impact of observation duration $\tau$ on the length of observation steps and available cooperative transmission rate. We set the average SNR $\bar{\gamma}$ to be 28,29 , and $30 \mathrm{~dB}$.

From Figure 10, we can see that the length of observation steps decreases slowly with the observation duration $\tau$ increases regularly. In other words, a larger $\tau$ results in a smaller number of observation steps. This is because the value of $\tau$ represents the cost of observing one SU candidate relay, and the PU needs to stop observing as soon as possible to avoid generating a large cost. On the contrary, when the value of $\tau$ is small, the cost for observation is low, and the PU tends to observe more candidate relays to find better cooperative relays. We also can see that different $\bar{\gamma}$ impacts the length of observation. A larger $\bar{\gamma}$ results

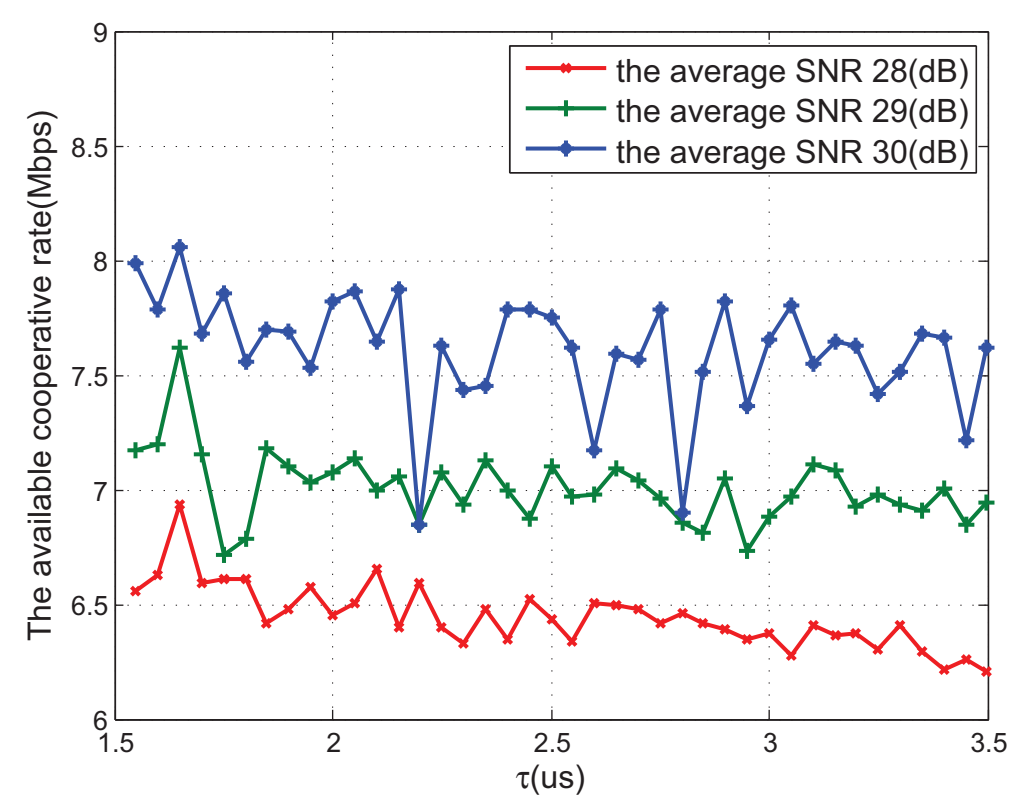

Figure 11 The available transmission amount vs. Time for one observation. 
in a larger the number of candidate relays according to Figure 7.

We can see from Figure 11 that the available cooperative transmission rate declines slowly with the increasing of the time duration for each observation $\tau$. This relationship can be obtained from the analysis in Section 4.2. When $\tau$ increases, the expected sum reward decreases. The decrease of the performance of selected relays, therefore, leads to a lower available cooperative transmission rate. On the other hand, the cost increases with the $\tau$ 's increasing. Therefore, the available cooperative transmission rate declines. Similarly, different $\bar{\gamma}$ impacts the available transmission rate. This observation is consistent with the analysis in Section 5.2 for Figure 7.

\section{Conclusions}

In this paper, we propose an optimal multiple stopping scheme to solve the problem of multiple cooperative relay selection for supporting smartphone-based HD video chat in CRNs. In the proposed scheme, the video source selects the candidate CR relays by following a proposed optimal multiple stopping rule to guarantee the success of HD video chat. The proposed scheme has been verified to be able to support $720 \mathrm{P}$ video chat via simulations. In the future, we will implement the proposed scheme on android smartphones.

\section{Competing interests}

The authors declare that they have no competing interests.

\section{Acknowledgements}

The authors would like to thank the support from the National Natural Science Foundation of China (Grant No. 61272505, 61172074 and 61371069) and the National Science Foundation of the US (CNS-1265311).

\section{Author details}

${ }^{1}$ School of Electronics and Information Engineering, Beijing Jiaotong University, No.3 Shangyuancun, 100044 Beijing, China. ${ }^{2}$ Department of Computer Science, Virginia Commonwealth University, 401 West Main Street, 23284 Richmond, VA, USA. ${ }^{3}$ Department of Computer Science, The George Washington University, 801 22nd Street NW, 20052 Washington DC, USA.

Received: 2 September 2014 Accepted: 9 February 2015

Published online: 03 March 2015

\section{References}

1. B Gonzalez, All about Internet speed requirements for Hulu, Netflix, and Vudu Movie Viewing. http://hometheater.about.com/od/ internethometheater2/a/Internet-Speed-for-Netflix-Vudu.htm

2. M Sullivan, 3G/4G performance map: data speeds for AT \& T Sprint, T-Mobile, and Verizon. http://www.techhive.com/article/254888/3g_4g_ performance_map_data_speeds_for_atandt_sprint_t_mobile_and_ verizon.html

3. J Jansen, P Cesar, DC Bulterman, T Stevens, I Kegel, J Issing, Enabling composition-based video-conferencing for the home. Multimedia IEEE Trans. 13(5), 869-881 (2011)

4. Y Lu, Y Zhao, F Kuipers, P Van Mieghem, in NETWORKING 2010. Measurement study of multi-party video conferencing (Springer-Verlag Berlin, Heidelberg, 2010), pp. 96-108

5. S Mirta, T Schierl, T Wiegand, P Inigo, C LeGuern, C Moreau, L Guarnieri, J Tronc, in Advanced Satellite Multimedia Systems Conference (asma) and the 11th Signal Processing for Space Communications Workshop (spsc). HD video broadcasting using scalable video coding combined with dvb-s2 variable coding and modulation (Cagliari, Italy, 2010), pp. 114-121

6. KR Rao, Do Nyeon Kim, in System Theory (SSST), 42nd Southeastern Symposium On. Current video coding standards: H. 264/avc, dirac, avs china and vc-1 (IEEE Tyler, TX, USA, 2010), pp. 1-8

7. H Schwarz, G Sullivan, T Tan, T Wiegand, in Circuits Syst. Video Technol. IEEE Trans. Comparison of the coding efficiency of video coding standards-including high efficiency video coding (HEVC), vol. 22, (2012), pp. $1669-1684$

8. H Schwarz, D Marpe, T Wiegand, Overview of the scalable video coding extension of the h.264/avc standard. Circuits Syst. Video Technol. IEEE Trans. 17(9), 1103-1120 (2007)

9. W Elmenreich, N Marchenko, H Adam, C Hofbauer, G Brandner, C Bettstetter, $\mathrm{M}$ Huemer, Building blocks of cooperative relaying in wireless systems. Elektrotechnik und Informationstechnik. 125, 353-359 (2008)

10. Z Cai, S Ji, J He, L Wei, AG Bourgeois, Distributed and asynchronous data collection in cognitive radio networks with fairness consideration. Parallel Distributed Syst. IEEE Trans. 25(8), 2020-2029 (2014)

11. $Y$ Jing, $H$ Jafarkhani, Single and multiple relay selection schemes and their achievable diversity orders. Wireless Commun. IEEE Trans. 8(3), 1414-1423 (2009)

12. G Amarasuriya, M Ardakani, C Tellambura, in Wireless Communications and Networking Conference (WCNC). Adaptive multiple relay selection scheme for cooperative wireless networks (IEEE Sydney, Australia, 2010), pp. 1-6

13. T Jing, S Zhu, H Li, X Cheng, Y Huo, in IEEE INFOCOM Mini-Conference. Cooperative relay selection in cognitive radio networks (Turin, Italy, 2013)

14. N Stratton, Reality of H.264 SVC. http://www.robotics.net/2013/02/04/ reality-of-h-264-svc/

15. W Su, AK Sadek, KR Liu, Cooperative communication protocols in wireless networks: performance analysis and optimum power allocation. Wireless Pers. Commun. 44(2), 181-217 (2008)

16. J Li, S Li, F Zhao, R Du, Co-channel interference modeling in cognitive wireless networks. Commun. IEEE Trans. 62(9), 3114-3128 (2014)

17. $\mathrm{S} \mathrm{Li}, \mathrm{G} \mathrm{Bi}$, Time domain averaging and correlation-based improved spectrum sensing method for cognitive radio. EURASIP J. Adv. Signal Process. 46(1), 520-532 (2014)

18. Y Chen, C-X Wang, H Xiao, D Yuan, Novel partial selection schemes for AF relaying in Nakagami-fading channels. Vehicular Technol. IEEE Trans. 60(7), 3497-3503 (2011)

19. HXiao, S Ouyang, Power control game in multisource multirelay cooperative communication systems with a quality-of-service constraint. Int. Trans. Syst. IEEE Trans. 16(1), 41-50 (2015)

20. Z Cai, J He, AG Bourgeois, S Ji, in 2012 IEEE 32nd International Conference On Distributed Computing Systems (ICDCS), Macau, China. Optimal distributed data collection for asynchronous cognitive radio networks, (2012), pp. 245-254

21. Z Cai, Y Duan, AG Bourgeois, Delay efficient opportunistic routing in asynchronous multi-channel cognitive radio networks. J. Combinatorial Optimization, 1-21 (2013)

22. G Bianchi, Performance analysis of the IEEE 802.11 distributed coordination function. Selected Areas Commun. IEEE J. 18(3), 535-547 (2000)

23. Y Duan, Z Cai, G Liu, Opportunistic channel-hopping based effective rendezvous establishment in cognitive radio networks. Wireless Algorithms Syst. Appl. 7405, 324-336 (2012)

24. M Hristakeva, D Shrestha, in The Midwest Instruction and Computing Symposium. Different approaches to solve the 0/1 knapsack problem (Eau Claire, WI, USA, 2005) 